\title{
A Transcrição de Exames na Consulta de Medicina Geral e Familiar: Perspetiva Ética Sobre o Caso do Pedido de Tomografia Computorizada Maxilofacial para Realização de Implantes Dentários
}

\section{Diagnostic Test Requests in the Family Medicine Consultation: An Ethical Perspective Concerning Requests for Maxillofacial Computerized Tomography Scan Prior to Dental Implants}

Paulo SANTOS $\square^{1,2}$

Acta Med Port 2019 Jun;32(6):415-418 - https://doi.org/10.20344/amp.11964

\begin{abstract}
RESUMO
A saúde oral tem sido pouco cuidada no contexto do Serviço Nacional de Saúde em Portugal. A quase inexistência de uma rede de medicina dentária levanta problemas de acessibilidade que condicionam a necessidade de complementar os serviços públicos com uma oferta predominantemente privada. Mas esta coexistência não é sempre fácil, sobretudo quando há necessidade dos serviços se cruzarem e colaborarem ativamente na resolução dos problemas do doente. Uma situação comum é a colocação de implantes dentários e a necessidade de realizar uma tomografia computorizada maxilofacial prévia para estudo de leito para colocação de implantes dentários osteointegrados, onde a aplicação de regulação governamental desenquadrada do contexto clínico pode condicionar a acessibilidade aos tratamentos. Com base neste cenário, discutem-se as opções do ponto de vista ético, na perspetiva do doente, do médico e da relação entre ambos. Conclui-se que a decisão médica não pode ser desenquadrada de uma avaliação clínica que apenas o ambiente de intimidade da consulta médica pode proporcionar. É um imperativo ético que se sobrepõe aos constrangimentos administrativos e burocráticos e que se bem gerido é potenciador de uma melhor saúde e de maior capacitação da pessoa.

Palavras-chave: Cuidados de Saúde Primários; Encaminhamento e Consulta; Implantes Dentários; Padrões de Prática Médica; Relações Médico-Doente; Sector Privado; Sector Público; Tomografia Computorizada
\end{abstract}

\section{ABSTRACT}

In the Portuguese National Health Service, little attention has been paid to oral health care. The almost nonexistence of a dentistry network raises concern about accessibility to services, and justifies the need to call on a predominantly private provision of services. The coexistence between the public and private settings is not always easy, especially when services need to interact and actively collaborate in order to find answers to the patient's problems. Dental implant procedures and the need to perform a previous maxillofacial computerized tomography to study the bone bed where osseointegrated dental implants are placed are a common situation. The current governmental regulation, blinded to the clinical context, may limit the accessibility to the tests. Based on this scenario, we discuss the possible options from an ethical point of view, framing the patient's and the physician's perspective and the relation between both. We conclude that the medical decision can't be disregarded from the clinical evaluation, in the intimacy of the medical consultation. This is an ethical duty that overrules the administrative and bureaucratic constraints. A good management of this apparent dichotomy may enhance better health and greater empowerment for the patient.

Keywords: Dental Implants; Physician-Patient Relations; Practice Patterns, Physicians; Primary Health Care; Private Sector; Public Sector; Referral and Consultation; Tomography, X-Ray Computed

Em Portugal, o acesso às consultas de Saúde Oral é deficitário e inequitativo, ${ }^{1}$ o que deriva da decisão de manter a estomatologia / medicina dentária dentro das instituições hospitalares e naturalmente orientada para o tratamento das doenças da boca e dentes, em vez de se organizar numa estrutura de proximidade mais própria dos Cuidados de Saúde Primários (CSP) e mais orientada para a promoção da saúde.

Apesar da tentativa dos últimos anos de melhorar a situação, nomeadamente com a publicação do Despacho n. ${ }^{0}$ 153/2005, que cria o Programa Nacional para a Promoção da Saúde Oral, com os sucessivos alargamentos, ainda não está garantida a satisfação das necessidades dos cidadãos na área da saúde oral. Uma parte significativa da população portuguesa não consegue aceder a consultas e tratamentos de saúde oral através do Serviço Nacional de Saúde (SNS). Os dados do Instituto Nacional de Estatística, em 2014 , mostram que cerca de $20 \%$ da população com 16 e mais anos de idade referiu necessidades não satisfeitas de consulta com dentista nos últimos 12 meses, sobretudo por falta de disponibilidade financeira.

Esta insuficiente resposta condiciona a necessidade de recorrer ao sector privado da saúde. Os dados do Barómetro da Saúde Oral, de 2017, da Ordem dos Médicos Dentistas, mostram que $78,3 \%$ dos portugueses continuam a pagar diretamente a consulta e tratamentos dentários, e

1. Departamento de Medicina da Comunidade, Informação e Decisão em Saúde (MEDCIDS). Faculdade de Medicina. Universidade do Porto. Porto. Portugal.

2. CINTESIS - Centro de Investigação em Tecnologias e Serviços de Saúde. Universidade do Porto. Porto. Portugal.

$\triangle$ Autor correspondente: Paulo Santos. psantosdr@med.up.pt

Recebido: 20 de fevereiro de 2019 - Aceite: 11 de abril de 2019 | Copyright @ Ordem dos Médicos 2019 
que os cheques-dentista representam apenas $0,2 \%,{ }^{2}$ num estudo em que $90 \%$ dos participantes não tentou sequer recorrer ao SNS.

É, portanto, comum em Portugal que um cidadão recorra a serviços prestadores privados para ver satisfeitas as suas necessidades de saúde na área da estomatologia e medicina dentária. Levanta-se a questão se será lícito ao cidadão pedir ao SNS o retorno da comparticipação sobre os procedimentos diagnósticos e os tratamentos efetuados. Especificamente na área da implantologia, onde é necessária a realização de exames prévios que oneram o procedimento, como a tomografia computorizada (TC) maxilofacial, será ético o cidadão recorrer aos serviços públicos de saúde para obter a comparticipação do exame? Será ético o médico recusar o pedido do exame com base em normas administrativas?

\section{$\mathrm{O}$ direito à saúde}

A Declaração Universal dos Direitos Humanos estabelece no seu artigo $25^{\circ}$ que toda a pessoa tem direito a um nível de vida suficiente para lhe assegurar, e à sua família, a saúde e o bem-estar, incluindo a alimentação, vestuário, alojamento, assistência médica, e serviços sociais necessários.

Este princípio foi plasmado no artigo $64 .^{\circ}$ da Constituição da República que garante o direito à saúde, através de um SNS "universal e geral e, tendo em conta as condições económicas e sociais dos cidadãos, tendencialmente gratuito". Ao Estado incumbe "garantir o acesso de todos os cidadãos, independentemente da sua condição económica, aos cuidados da medicina preventiva, curativa e de reabilitação," e articular com o SNS as formas empresariais e privadas da medicina, assegurando a eficiência e a qualidade da prestação.

Podemos afirmar que, em Portugal, a Saúde é um direito de todos os cidadãos através do SNS, público, que se articula com entidades privadas para garantir o acesso aos cuidados médicos, no chamado sistema nacional de saúde.

Não sendo específica na definição da carteira de serviços acessíveis, presume-se que a globalidade de cuidados, constitucionalmente definida, integre a totalidade de necessidades de saúde dos cidadãos, decorrentes dos seus legítimos interesses.

Assim, é lícito aos cidadãos procurar resposta às suas necessidades de saúde fora do SNS, sobretudo quando este não garante o acesso como é o caso de grande parte dos tratamentos de Estomatologia e Medicina Dentária. Numa perspetiva de complementaridade de cuidados, assumindo o direito à saúde como universal e geral, é também função dos serviços públicos providenciar os requisitos necessários à satisfação das necessidades de saúde legitimamente identificadas.

\section{Legitimidade para o pedido do teste diagnóstico}

A primeira questão coloca-se ao nível da necessidade da realização deste exame de diagnóstico, por se registar alguma variabilidade na decisão.
A colocação de implantes dentários não é decisão da consulta do médico generalista, nem a sua especificidade ou os constrangimentos associados o são.

A leges artis resumida pelas orientações aceites nesta área de intervenção aponta para a necessidade de realizar um estudo prévio através da TC maxilofacial. Em Portugal, a Direção Geral da Saúde emitiu a Norma de Orientação Clínica n. ${ }^{\circ}$ 049/2011, de 27/12/2011, onde refere que a sua realização está indicada na "avaliação dos rebordos ósseos alveolares, para estudo de leito para colocação de implantes dentários osteointegrados, em particular quando é necessário identificar estruturas anatómicas adjacentes (por exemplo, seio maxilar, nervo dentário inferior), a definição da forma anatómica do osso ou a densidade óssea" (Grau de recomendação I, Nível de evidência C), entre outras indicações.

\section{Legitimidade para recorrer ao médico assistente}

Não se colocam dúvidas sobre a legitimidade de o cidadão recorrer a um serviço privado de prestação de cuidados de saúde oral. A questão coloca-se quanto ao facto de recorrer ao seu médico assistente nos CSP para ver satisfeita uma condição necessária à prossecução do tratamento proposto por médico devidamente habilitado e aceite pela pessoa.

Na realidade, não está em causa a realização do exame pela pessoa pois a sua prescrição pelo médico de especialidade é válida desde que enquadrada nas normas deontológicas publicadas pelas respetivas Ordens Profissionais. O que está em causa é o acesso aos serviços de saúde na medida em que o exame em causa será comparticipado se prescrito através de credencial própria do SNS e não será se utilizado outro qualquer formulário de prescrição, fora do contexto dos sistemas complementares de saúde.

Identificado o problema em causa e assumindo que os CSP, através dos médicos de Medicina Geral e Familiar (MGF), têm por missão a prestação de cuidados de saúde personalizados à população inscrita de uma determinada área geográfica, garantindo a acessibilidade, a globalidade, a qualidade e a continuidade dos mesmos (Dec.-Lei n. ${ }^{\circ}$ 298/2007, com as alterações introduzidas pelo Dec.-Lei n. ${ }^{\circ} 73 / 2017$ ), é lícito ao cidadão procurar no seu médico de família a solução para o seu problema de acessibilidade.

\section{Legitimidade para o médico transcrever a prescrição}

É entendimento da tutela administrativa que não deve haver transcrições cruzadas de pedidos de exames entre os diferentes níveis de cuidados assistenciais. O Despacho n. ${ }^{\circ}$ 10430/2011, de 1 de agosto de 2011, do Secretário de Estado da Saúde, proíbe expressamente no seu ponto 3 a solicitação da prescrição de exames às unidades de CSP pelos estabelecimentos hospitalares integrados no SNS, pelas unidades convencionadas de hemodiálise, pelos hospitais privados e pelos médicos no exercício de medicina privada, e a sua transcrição pelos médicos dos CSP.

Mas quem pede a transcrição não é de facto a instituição, mas o cidadão, que pretende o acesso a um exa- 
me necessário à resolução de um problema de saúde que identifica como relevante no seu contexto.

A decisão depende do contexto e não do exame ou de quem o requisita, e é no contexto que se avalia a situação em concreto e se ajusta a decisão clínica. ${ }^{3}$ Importa perceber a adequação do diagnóstico, da proposta terapêutica e da sua implicação prognóstica, e isso implica uma avaliação clínica ponderada pelos princípios da ética médica na intimidade da consulta. Os exames auxiliares de diagnóstico ou terapêutica são enquadrados nesta avaliação clínica e devem responder a questões específicas, ${ }^{4}$ permitindo melhorar a efetividade diagnóstica e terapêutica para uma prática baseada na melhor evidência. ${ }^{5}$

\section{De quem são os exames?}

Classicamente a medicina desenvolveu-se numa lógica de um médico ativo que propõe ao doente passivo a melhor opção, dada a avaliação realizada. Este modelo paternalista evoluiu para um modelo de decisão partilhada onde o doente assume uma maior importância nas suas expectativas, nos seus valores e nas suas opções. ${ }^{6}$

Este posicionamento aumenta a responsabilidade do cidadão, para uma autonomia em que, de uma forma livre, mas esclarecida, decide em cada momento a sua própria opção no que à saúde diz respeito.

Deriva deste princípio que é o cidadão quem dispõe da sua informação de saúde, e do poder de decisão sobre ela, desde a escolha do médico assistente, até aos sintomas que refere num dado contexto, às manobras do exame objetivo a que se sujeita e aos meios auxiliares de diagnóstico que irá realizar.

Os resultados dos testes realizados pertencem ao cidadão que deles fará o que melhor lhe aprouver, ${ }^{7,8}$ tendo em conta a bidirecionalidade do modelo, em que só poderá esperar o melhor resultado em saúde se permitir ao médico o acesso à informação necessária para a construção da melhor decisão clínica.

\section{Qual é então o papel do médico?}

$\mathrm{O}$ ato médico é um conjunto de procedimentos que levam ao estabelecimento de um diagnóstico, proposta terapêutica (com intenção preventiva, curativa ou de reabilitação) e respetivo prognóstico. A International Standard Classification of Occupations da Organização Internacional do Trabalho (ISCO-08) operacionaliza esta definição indicando o médico como aquele que diagnostica e trata doenças, perturbações de saúde e traumatismos em seres humanos, e recomenda ações preventivas, baseado nos princípios científicos da medicina moderna.

No seu exercício, técnica e deontologicamente independente, o médico obriga-se responsavelmente pela prestação dos melhores cuidados ao seu alcance, com correção e delicadeza, no intuito de promover ou restituir a saúde, conservar a vida e a sua qualidade, e suavizar os sofrimentos, no pleno respeito pela dignidade do ser humano.

No seu compromisso ético, o médico assume como primeira preocupação a saúde e o bem-estar da pessoa que o procura, respeitando a sua autonomia e sua dignidade, e não permitindo que outra qualquer consideração se interponha no seu dever e entre si e o seu doente.

Numa situação em que a pessoa, portadora de uma prescrição de um tratamento ou de um exame, procura os cuidados médicos para a resolução de um problema de acessibilidade, cabe ao médico (aqui médico de família) avaliar pelo respeito dos princípios éticos da beneficência, da não maleficência, da justiça, da verdade, da confidencialidade, e, com respeito pela autonomia do doente, decidir em consciência e dignidade e de acordo com as boas práticas, ${ }^{9}$ ainda que não esteja diretamente envolvido na avaliação que deu origem à prescrição, ou na orientação que se seguirá. Integrar as expectativas do doente, as suas opiniões e os seus valores numa decisão clínica estruturada e participada é uma atitude comum no exercício em MGF, seja na decisão ao nível dos CSP, ou quando o médico é chamado a opinar sobre o processo do doente que se encontra referenciado a outras especialidades, reforçando, mantendo, ou, por vezes, argumentando em discordância com outras interpretações da realidade. Ao invés de claudicar na controvérsia, criam-se oportunidades de educação geradoras de mais e melhor saúde. ${ }^{10}$

\section{Conclusão}

A necessidade de comunicação entre médicos de diferentes níveis de cuidados é tão antiga quanto a diferenciação das especialidades. A introdução do conceito de autonomia dos cidadãos veio aprofundar esta necessidade e ajudar a clarificar os procedimentos, garantindo que é ao cidadão, doente ou em saúde, que compete a opção no que à sua saúde diz respeito.

Este princípio alarga-se quando o doente assume uma dificuldade no percurso dentro do sistema de saúde e recorre ao seu médico assistente, mais frequentemente nos CSP, para a sua orientação.

Neste contexto, podemos concluir que não se verificam constrangimentos de natureza ética suscetíveis de colocar em risco a relação do cidadão com o seu médico assistente na opção que faz por um determinado tratamento, no caso a colocação de implantes dentários, nem no facto de a ele recorrer para um problema que, não tendo a ver diretamente com esta opção, está relacionado com o acesso a um meio auxiliar diagnóstico considerado relevante para a concretização do procedimento.

Na garantia do princípio basilar de equidade no acesso, é obrigação ética do médico procurar enquadrar uma resposta que apresente uma racionalidade baseada na melhor evidência, integrando a sua própria experiência clínica e as expectativas e opiniões do doente, ${ }^{11}$ e da comunidade envolvente.

Não há justificação para que um determinado enquadramento administrativo se sobreponha aos princípios éticos do exercício médico, pelo que a decisão de transcrever ou não o pedido de exame num formulário que dê acesso à comparticipação pelo sistema de saúde público ou privado terá de ser circunstanciada numa ponderação técnica, deontológica e ética apenas possível na análise de 
cada caso específico, criando oportunidades de educação potenciadoras de uma melhor saúde.

Ao permitir a interferência externa de normativos desenquadrados da especificidade da situação clínica e social, a recusa liminar do pedido coloca em risco a relação

\section{REFERÊNCIAS}

1. Simões A, Paquete A, Araújo M. Equidade horizontal no acesso a consultas de clínica geral, cardiologia e medicina dentária em Portugal. Rev Port Saude Publica. 2008;26:39-52.

2. Ordem dos Médicos Dentistas. Barómetro da Saúde Oral. [consultado 2018 dez 03]. Disponível em: https://www.omd.pt/content/uploads/ 2017/12/barometro-saude-oral- 2017.pdf2017.

3. Hundert EM. "Ought” implies "can”. Harv Rev Psychiatry. 1994;1:301-2.

4. Whiting P, Toerien M, de Salis I, Sterne JA, Dieppe P, Egger M, et al. A review identifies and classifies reasons for ordering diagnostic tests. J Clin Epidemiol. 2007;60:981-9.

5. McCormack JP, Loewen P. Adding "value" to clinical practice guidelines. Can Fam Physician. 2007;53:1326-7.

6. Taylor K. Paternalism, participation and partnership - the evolution of patient centeredness in the consultation. Patient Educ Couns. 2009;74:150-5. médico-doente, e pode condicionar um prejuízo significativo para o doente e para o regular funcionamento das instituições, eventualmente superior ao que o legislador pretenderia evitar.

7. Keinert TM, Cortizo CT. Dimensões da privacidade das informações em saúde. Cad Saude Publica. 2018;34:e00039417.

8. Ventura M, Coeli CM. Para além da privacidade: direito à informação na saúde, proteção de dados pessoais e governança. Cad Saude Publica. 2018;34:e00106818.

9. Savulescu J, Schuklenk U. Doctors have no right to refuse medical assistance in dying, abortion or contraception. Bioethics. 2017;31:162-70.

10. Santos $P$, Hespanhol A. An ethical view of vaccine refusal. Rev Port Med Geral Fam. 2013;29:328-33.

11. Sackett DL, Straus SE, Richardson WS, Rosenberg W, Haynes RB. How to practice and teach EBM. Edinburgh: Churchill Livingstone; 2000. 